\title{
SUSCEPTIBILIDADE DE BIOMPHALARIA TENAGOPHILA (d'ORBIGNY, 1835), DE ITAJUBÁ (MG), À INFECÇÃO PELA CEPA "LE" DE SCHISTOSOMA MANSONI SAMBON, 1907, DE BELO HORIZONTE, MG (BRASIL) *
}

Omar dos Santos Carvalho **

Roberto Milward-de-Andrade ***

Cecilia Pereira de Souza**

RSPUB9/442

Carvalho, O. dos $\mathrm{S}$. et al. Susceptibilidade de Biomphalaria tenagophila (d'Orbigny, 1835), de Itajubá $(M G)$, à infecção pela cepa "LE" de Schistosoma mansoni Sambon, 1907, de Belo Horizonte, MG (Brasil). Rev. Saúde públ., S. Pallo, $13: 20-5,1979$.

RESUMO: Infecções experimentais de descendentes de Biomphalaria tenagophila de Itajubá, $M G$ (Brasil) foram realizadas utilizando-se a conhecida cepa " $L E$ " de Schistosoma mansoni, isolada em Belo Horizonte. Como controle, utilizou-se B. glabrata de Belo Horizonte, $M G$. Em conseqüência, assinalou-se, pela primeira vez, a infeçāo de $\mathrm{B}$. tenagophila de Itajubá, à cepa de $\mathrm{S}$. mansoni adaptada $\grave{a} \mathrm{~B}$. glabrata de Belo Horizonte. Isto é, ao final do experimento, 5 (3,3\%) entre os 149 exemplares sobreviventes, infectaram-se. Face ao atual surto industrial da região, com grande aporte de migrantes, foi chamada a atenfão para a necessidade de medidas profiláticas, em decorrência da possibilidade de instalação de foco da doença no Sul de Minas Gerais.

UnITERmos: Esquistossomose mansônica. Biomphalaria Tenagophila, infecção experimental. Biomphalaria glabrata. Schistosoma mansoni.

\section{NTRODUCÃO}

A susceptibilidade da Biomphalaria tenagophila à infecção pelo Schistosoma mansoni era, até pouco tempo atrás, pouco compreendida. Considerava-se, mesmo, aquele molusco refratário ou, quando não, mau hospedeiro intermediário do trematódeo em questão - o que levou Lutz (1923) a denominá-lo, inclusive, de Planorbis immunis, obscurecendo, pois, durante longo tem- po, uma melhor compreensão de seu papel na epidemiologia da esquistossomose mansoni, em nosso meio.

A descoberta de focos ativos da helmintose em certas localidades do Estado de São Paulo, onde o planorbídeo existente era a $B$. tenagophila, constituiu ponto de partida para melhor compreensão do problema biológico envolvido, ou seja, reco-

* Trabalho complementado com auxílio do Conselho Nacional de Desenvolvimento Científico e Tecnológico (CNPq).

* Do Centro de Pesquisas "René Rachou"/FIOCRUZ. Av. Augusto de Lima, 1715. Caixa Postal 1743 - 30000 - Belo Horizonte, MG - Brasil.

** Do Centro de Pesquisas "René Rachou"/FIOCRUZ e do Departamento de Parasitologia da UFMG - Belo Horizonte, MG - Brasil. 
CARVAlHo, $O$. dos $S$. et al Susceptibilidale de Biomphalaria tenagophila (d'Orbigny, 1835), de Itajubá (MG), à infecção pela cepa "LE" de Schistosoma mansoni Sambon, 1907, de Belo Horizonte, MG (Brasil). Rev. Saúde públ., S. Paulo, 13:20-5, 1979.

nhecimento da especificidade do molusco à infecção por certas cepas do parasita e vice-versa. Este problema, em última análise, envolve questões relacionadas à especiação do trematódeo (Coelho, ${ }^{\text {6.i }}$ 1957, 1962; Ruiz,23 1957; Paraense e col.13,14.17 1963, 1964; Magalhães,0.10 1969, 1970 ; Chieffi, 5 1975; Borda e Pellegrino, 1976: Carvalho e col.,2.3 1977, 1978).

A partir de então, autores como Paraense e Deslandes 15, 1956; Piza e col.,19.20 1959, 1960; Ramos e col.21, 1961; Magalhães e col, ${ }^{11}$ 1973, entre outros, têm observado populações de $B$. tenagophila com altas taxas de infecção natural por $S$. mansoni, porém apenas no Estado de São Paulo.

No presente trabalho apresenta-se o resultado da exposição de $B$. tenagophila, proveniente da cidade de Itajubá (MG), à miracidios da cepa "LE" de S. mansoni.

Estudos morfológicos de exemplares dessa espécie (Australorbis nigricans), capturados na localidade em questão, foram realizados por Paraense e Deslandes ${ }^{16}$ (1955).

A localidade mencionada encontra-se no sul do Estado de Minas Gerais, sendo banhada pelo Rio Sapucaí, distando cerca de $430 \mathrm{Km}$, por rodovia de Belo Horizonte e cerca de $260 \mathrm{Km}$ de São Paulo, SP.

Assinala-se, ademais, que se trata de região até agora considerada indene de esquistossomose mansoni.

\section{MATERIAL E METODOS}

Os planorbideos utilizados descendiam de uma amostra coletada nos arredores da Faculdade de Medicina de Itajubá (Figura), enquanto us miracidios — obtidos segundo técnica descrita por Chaia * (1956) - pertenciam à cepa "LE" de S. mansoni, isolada em Belo Horizonte (Pellegrino e Katz ${ }^{18}$, 1968).

Como controle, utilizuu-se $B$. glabrata descendente de uma cepa originária do Barreiro de Cima, Belo Horizonte, MG.

Para infecção en massa dos planorbideos, utilizou-se água desclorada, em quantidade suficiente para recobrir os moluscos contidos em cristalizadores. Nestas condições, miracidios e caramujos eram mantidos por cerca de 15 hs., sob iluminação artificial.

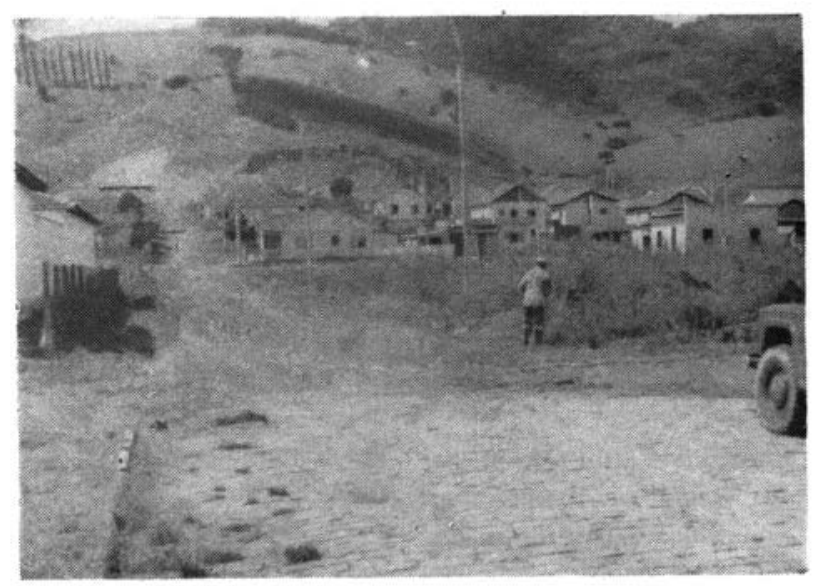

Vista da área adjacente à Faculdade de Medicina de Itajubá, MG (Brasil) onde se capturou grande número de Biomphalaria tenagophila. (29/3/1977). 
CARVALHO, O. dos $\mathrm{S}$. et al Susceptibilidaie de Biomphalaria tenagophila (d Orbigny, 1835), de Itajubá (MG), à infeç̧ão pela cepa "LE" de Schistosoma mansoni Sambon, 1907, de Belo Horizonte, MG (Brasil). Rev. Saúde públ., S. Paulo, 13:20-5, 1979.

\section{T A B E L A}

Índices de infecção experimental de Biomphalaria tenagophila capturadas em Itajubá (MG). expostas à miracídios de Schistosoma marasoni de Belo Horizonte. (Belo Horizonte, MG. Dez./76-out./77).

\begin{tabular}{|c|c|c|c|c|c|c|}
\hline \multirow[b]{2}{*}{ Grupo } & \multicolumn{3}{|c|}{ Infeç̧ão com S. mansoni } & \multicolumn{3}{|c|}{ Planorbídeos } \\
\hline & & & & & & \\
\hline 1 & $28 /$ dez. & 25 & 500 & 17 & 0 & - \\
\hline 2 & $"$ & 25 & 1.250 & 16 & 0 & $\rightarrow$ \\
\hline 3 & 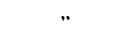 & 25 & 2.500 & 18 & 0 & 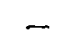 \\
\hline 4 & $13 /$ set. & 25 & 3.750 & 19 & 1) & - \\
\hline 5 & $"$ & 25 & 5.000 & 21 & 3 & 14.2 \\
\hline 6 & $"$ & 25 & 6.250 & 19 & 11 & - \\
\hline 7 & $"$ & 25 & 7.500 & 17 & 1 & 5,9 \\
\hline 8 & $"$ & 25 & 8.750 & 22 & 1 & 4.5 \\
\hline Controle** & & & & & & \\
\hline B. glabrata & 28/dez. & 60 & 1.200 & 21 & 19 & 90,5 \\
\hline B. glabrata & $13 /$ set. & 60 & 1.200 & 40 & 40 & 100,0 \\
\hline
\end{tabular}

* B. tenagonhila: após o 64 v dia da infecção.

* Coutrole: após 84 e 64 dias, respectivamente, da infecção.

Decorrido este período, os caramujos foram transferidos para bacias plásticas contendo água, proveniente de fonte natural, e areia, como substrato. A alimentação consistia de alface fresca, oferecida diariamente.

Os planorbíneos foram examinados individualmente por exposição à luz artificial.

Experimento 1 . Um total de 150 exemplares de B. tenagophila, com $15 \mathrm{~mm}$ de diâmetro, divididos em 6 grupos de 25 exemplares, foram expostos à 20,50,100,150, 200 e 250 miracídios/caramujo.

Como controle, utilizou-se 50 exemplares de $B$. glabrata, com $15 \mathrm{~mm}$ de diâmetro e infectados com $20 \mathrm{miracidios} / \mathrm{caramujo}$ da mesma cepa "LE" de S. mansoni.

As infecções foram feitas a 28 de dezembro de 1976 e os exames realizados 39 e 52 dias após.
Experimento 2. Um total de 200 exemplares de $B$. tenagophila com 5-7 mm de diâmetro, divididos em 8 grupos de 25 cada, foram expostos à infecção com 20,50,100, 150 , $200,250,300$ e $350 \mathrm{miracídios} /$ caramujo.

Como controle, utilizou-se $120 \mathrm{~B}$. glabrata $(5-7 \mathrm{~mm})$ divididos em 2 grupos de 60 exemplares. Todos foram submetidos à infecção com 20 miracídios/caramujo, da cepa "LE" de S. mansoni.

A 18 de agosto/77, infectou-se 3 grupos de $B$. tenagophila e um grupo controle. examinando-se $42,52,63$ e 84 dias após a infecção.

$\mathrm{Na}$ segunda etapa do experimento, os 5 grupos restantes foram infectados, juntamente com outro grupo controle, em 13/9/77 e examinados após $38,43,52$ e 64 dias da infecção. 
CARVALHO, $O \cdot d o s$ S. et al SusceptibilidaAe de Biomphalaria tenagophila (d'Orbigny, 1835), de Itajubá (MG), à infecção pela cepa "LE" de Schistosoma mansoni Sambon, 1907, de Belo Horizonte, MG (Brasil), Rev. Saude puibl., S. Paulo, 13:20-5, 1979.

\section{RESULTADOS}

Experimento 1. Os exames dos $145 B$ Tenagophila sobreviventes, realizados a 39 e 52 dias da infeç̧ão, não revelaram exemplares positivos.

No grupo controle, $15(60 \%)$ dos 25 exemplares de $B$. glabrata sobreviventes encontravam-se positivos para $S$. mansoni ao final do experimento.

A temperatura da água, durante o transcorrer do experimento, variou de $23^{\circ} \mathrm{C}$ a $26^{\circ} \mathrm{C}$.

Experimento 2. Os 3 primeiros grupos de $B$. tenagophila examinados, com 42, 52, 63 e 84 dias da infecção mostraram-se negativos (Tabela).

No grupo controle, $19(90,5 \%)$, dos 21 exemplares de $B$. glabrata sobreviventes, eliminavam cercárias de $S$. mansoni.

Nos 5 grupos restantes, examinados com $38,43,52$ e 64 dias após a infecção, encontrou-se $5(3,3 \%) \quad B$. tenagophila positivos para $S$. mansoni.

No grupo infectado com 200 miracídois/ /caramujo, 3 exemplares $(4,2 \%)$ de $B$. tenagophila revelaram-se positivos. Um exemplar ao $38^{\circ}$ dia; e dois outros, no $52^{\circ}$ dia da infecção.

No grupo infectado com 300 miracídios/ /caramujo, verificou-se que $5,9 \%$ (1) dos 17 sobreviventes eliminavam cercárias de S. mansoni. E, dentre 22 exemplares sobreviventes, submetidos a 350 miracídios/ /caramujo, 4,5\% (1) estavam positivos.

No grupo controle, $100 \%$ dos $40 \mathrm{~B}$. glabrata sobreviventes, no $38^{\circ}$ dia do período experimental, eliminavam cercárias de S. mansoni.

Durante o experimento a temperatura variou de $24^{\circ} \mathrm{C}$ a $25^{\circ} \mathrm{C}$.

\section{DISCUSSÃO}

Os resultados obtidos revelaram que : Biomphalaria tenagophila de Itajubá, MG mostrou-se susceptivel à bem conhecida cepa "LE" de Schistosoma mansoni isolada de paciente de Belo Horizonte, MG.

Tais dados diferem, pois, de pesquisa conduzida por Coelho' (1962), na qual $B$. tenagophila daquela localidade não se infectou com uma cepa do trematódeo de Belo Horizonte - no caso, na proporção de 10-20 miracídios para cada um dos 25 planorbídeos então utilizados.

Estudos de Paraense e Correa 1: (1963), demonstraram a existência no Vale do Paraiba (SP), de uma cepa de $S$. mansoni fisiologicamente adaptada à cepa simpátrica de $B$. tenagophila - porém, incapaz de infectar $B$. glabrata altamente susceptivel ao trematódeo de Belo Horizonte.

Para esses mesmos autores (1963), existe no Vale do Paraíba uma cepa mutante de $S$. mansoni pré-adaptada à $B$, tenagophila que, entretanto, continuaria refratária ao trematódeo ancestral. Os esquistossomos originários da hibridação: cepa Vale do Paraiba $\mathrm{x}$ cepa de Belo Horizonte, afirmam os autores, apresentam comportamento diverso das cepas originais de $S$. mansoni, quanto à susceptibilidade aos planorbídeos $B$. tenagophila e $B$. glabrata.

Magalhães (1970), sugere a existência de miracidios com "genótipo pré-adaptado às condições fornecidas pelo genótipo do molusco", entre as populações utilizadas em infecções experimentais e nas quais têm sido obtidas baixas taxas de infecção como é, por exemplo, o caso de $B$. tenagophila do Vale do Paraíba submetidas à infecção com S. mansoni de Belo Horizonte.

Por outro lado, pode-se observar uma variabilidade na taxa de infecção face ao diâmetro dos moluscos, isto é, os exemplares menores (5-7 $\mathrm{mm})$ mostraram-se mais susceptiveis que os de maior porte (15 $\mathrm{mm})$ utilizados no experimento 1 . Esses resultados concordam com os achados de outros autores (Newton ${ }^{12}$, 1953; Wright 26, 1963; Richards 22, 1973; Santana e col.24, 1978). Contudo, Sturrock e col.25 (1970), não observaram qualquer evidência de redução na susceptibilidade de $B$. gla- 
CARVALHO, O. dos $\mathrm{S}$. et al Susceptibilidade de Blomphalaria tenagophla (d'Orbigny, 1835), de Itajubá (MG), à infecção pela cepa "LE" de Schistosoma mansoni Sambon, 1907, de Belo Horizonte, MG (Brasil), Rer. Saúde pribl., S. Paulo, 13:20-5, 1979.

brata de Santa Lúcia (Indias Ocidentais), devido ao diâmetro dos moluscos, quando esses estão bem adaptados à cepa do trematódeo.

O encontro ora relatado enfatiza a necessidade de atenção especial para o problema, considerando-se principalmente o surto industrial na área de Itajubá, com significativo aporte atual de migrantes inclusive de tradicionais focos de trans- missão devidos à presença de S. mansoni, possivelmente, adaptado à cepa local de $B$. tenagophila.

En outros termos, se adequadas medidas profiláticas não forem tomadas, aquela área corre o risco de tornar-se mais um foco da doença. $\mathrm{Na}$ verdade, seria o início da introdução e fixação de foco endêmico no sul do Estado de Minas Gerais, região até agora indene.

CARVAl.HO, O. dos S, et al. /The susceptibility of the Biomphalaria tenagophila (d'Orbigny, 1835) from Itajuba, Minas Gerais to infection by' strain "LE" of the Schistosoma mansoni (Sambon, 1907) from Belo Horizonte. Minas Gerais, Brazil.] Rev. Saúde públ., S. Pallo, 13: - . 1979.

ABSTRACT: Descendents of Biomphalaria tenagophila from Minas Gerais, Brazil were experimentally infected with the Schistosoma mansoni. B. glabrata from Belo Horizonte was used as control. The "LE" strain of S. mansoni used in both cases was isolated in Belo Horizonte. B. tenagophila from Itajuba was shown, for the first time, to be susceptible to the strain of S. Mansoni adapted to B. glabrata from Belo Horizonte. A $3.3 \%$ infection rate was observed, i.e., 5 of the I 49 specimen which survived until the end of the experiment were infected.

UNITERHS: Schistosomiasis. Biomphalaria tenagophila, experimental infection. Biomphalaria glabrata. Schistosoma mansoni.

\section{REFERENCIAS BIBLIOGRAFICAS}

1. BORDA. C. E. \& PELLEGRINO J. Susceptibilidade de Biomphalaria tenagophla * B. glabrata a dos cepas de Schistosoma mansoni. Rev. Inst. Med. trop S Paulo, 18:157-64, 1976.

2. Carvalio, O. S. et al. Infecção expemental de Bromphalaria tenagophila (d'Orbigny. 1835) de Itajubá (MG, Brasil), à cepa "LE" de Schistosoma mansoni Sambon. 1907. In: Congresso da Sociedade Brasileira de Medicina Tropical. 14" e Congresso da Sociedade Brasileira de Parasitologia. 29, 1978. João Pessoa. 1978. p. $1 / 51$.

3. CarvalHo, O. S. et al. Susceptibilidade de Biomphalaria tenagophila (d'Orbigny, 1835) de Itajubá (MG, Brasil) à repa "LE" de Schistosoma manson Sambon, 1907 Cienc. Cult., 29:786. 1977.

4. CHAIA, G. Técnica para a concentração de miracídios. Rer. bras. Malar., 8:355-7. 1956 .

5. CHIEFfi. P. P. Susceptibilidade à infecção por Schistosoma manson, de cepas de Biomphalaria tenagophila originários dos Estados de São Paulo e Paraná. Rer. Inst. Med. trop. S. Paulo, 17:92-6, 1975.

6. COELHO, M. V. Aspectos do desenrolvimento das formas larvais do schistosoma manson em Australorbis nigricans. Rer. bras. Biol., 17:325-37. 1957. 
CARVAlHo, o. dos $\mathrm{S}$. et al Susceptibilidade de Biomphalaria tenagophila (d'Orbigny, 1835), de Itajubá (MG), à infecção pela cepa "LE" de Schistosoma mansoni Sambon, 1907, de Belo Horizonte, MG (Brasil). Rev. Saúde públ., S. Paulo, 13:20-5, 1979.

7. COELHo, M. V. Susceptibilidade de Australorbis tenagophila à infecção por Schistosoma mansoni. Rev. Inst. Med. trop. S. Paulo, 5:289-95, 1962.

8. LUTZ, A. Planorbis immunis n.n. Nautilus, 37:36, 1923.

9. MAGALHães, L. A. Estudo dos dados obtidos de uma população de Biomphalaria glabrata de Belo Horizonte infectada por Schistosoma mansoni da mesma cidade, e de uma população de $B$. tenagophila de Campinas, infectada por S. mansoni de São José dos Campos. Rev. Soc. bras. Med. trop., 3: 195-6, 1969.

10. MAGALHAES, L. A. Estudo do comportamento da cepa de $S$. mansoni de Brasília. Hospital, Rio de Janeiro, 77: $669-78,1970$.

11. MAGALHÃE, L. A. et al. Aspectos epidemiológicos dá esquistossomose mansônica na região da represa de Americana, Estado de São Paulo, Brasil. Rev. Saude puibl., S. Paulo, 7:21-8. 1973.

12. NEWTON, $W$. $L$. The inheritance of susceptibility to infection with Schistosoma mansoni in Australorbis glabratus. Exp. Parasit., 2:242-57, 1973.

13. PARAENSE, W, L. \& CORREA, L. R. Sobre a ocorrência de duas raças biológicas do Schistosoma mansoni no Brasil. [Apresentado à XV Reunião Anual da Sociedade Brasileira para o Progresso da Ciència, Campinas, 1963.]

14. PARAENSE, W. L. \& CORREA, L. R. Susceptibility of Australorbis tenagophilus to infection with Schistosoma mansoni. Rev. Inst. Med. trop. S. Paulo, 5:23-9, 1963.

15. PARAENSE, W. L. \& DESLANDES, N. Australorbis nigricans as the transmitter of schistosomiasis in Santos. state of São Paulo. Rev. bras, Malar., $8: 235-45,1956$.

16. PARAENSE, W. L. \& DESLANDES, N. Observations on the morphology of Australorbis nigricans. Mem. Inst. Oswaldo Cruz, 53:121-34, 1955
17. PARAENSE, W. L. et al, Australorbis tenagophilus in Peru, and susceptibility to Schistosoma mansoni. Amer. $J$. trop. Med. Hyg., 13:534-40, 1964.

18. PELEGRINO, J. \& KATZ, N. Experimental chemoterapy of schistosomiasis mansoni. Advanc. Parasitol., 6:23390, 1968.

19. PIZA, J, T. et al. A esquistosomose no Vale Paraiba. Rev. Inst. Adolfo Lutz, $19: 97-143,1959$.

20. PIZA, J. T. et al. Vale do Paraiba (Estado de São Paulo - Brasil). Importância epidemiológica do Taphius nigricans (Spix, 1827). Arq. Hig., S. Paulo, $25: 35-40,1960$.

21. RAMOS, A. S. et al. Observaçoes sobre Australorbis tenagophilus, transmissor da esquistossomose mansônica. Arq. Hig., S. Paulo, 26:121-4, 1961.

22. RICHARDS. C, S. Susceptibility of adult Biomphalaria glabrata to Schistosoma mansoni infection. Amer. J. trop. Med. Hyg., 22:748-56, 1973.

23. RUIZ, J. M. Esquistossomose experimental. 5. Dados sobre a infestação experimental de Biomphalaria tenagophila (Orbigny) e Australorbis glabratus (Say), Rev, bras, Biol., 17:179-85, 1957.

24. SANTANA, J. V. et al. Seleção de linhagens de Biomphalaria tenagophila e Biomphalaria glabrata visando major suscetibilidade ao Schistosoma mansoni. Rev. Saúde publ., S. Paulo, 12: 67-77, 1978 .

25. STURROCK, B. M. \& STURROCK, R. F. Laboratory studies of the host-parasite relationship of Schistosoma mansoni and Biomphalaria glabrata from St. Lúcia, West Indies. Ann trop. Med. Parasit., 64:357-63, 1970.

26. WRIGHT, C. A. \& ROSS, G. C. Eletrophoretic studies of blood and egg proteins in Australorbis glabratus (Gastropoda, Planobidae), Ann. trop. Med. Parasit, $57: 47-51,1963$.

Recebido para publicasão em 05/06/1978 Aprovado para publicaça em e9/08/1978 